\title{
Momentum Balance in Zonal Flows and Resonance of Baroclinic Rossby Waves*
}

\author{
ChRISTOPH VÖLKER ${ }^{+}$ \\ Alfred-Wegener-Institut für Polar- und Meeresforschung, Bremerhaven, Germany
}

(Manuscript received 2 January 1998, in final form 21 July 1998)

\begin{abstract}
Wind-driven flow in a baroclinic quasigeostrophic channel with simple bottom topography is studied in a model with reduced physics and degrees of freedom as an analogy to the Antarctic Circumpolar Current. For a sinusoidal topography an approximate analytical solution is found using a low-order spectral model. Resonance of baroclinic Rossby waves can lead to different flow regimes of which one is a blocked state, where most of the momentum, imparted to the fluid by the wind stress, is transferred to the earth by bottom form stress. For some parameter values there are both resonant and nonresonant solutions to the model equations. It is shown that these results of the low-order model apply also to a more complicated spectral model with sinusoidal but also with Gaussian ridge topography. The steady states of these models are found numerically using a continuation algorithm. In the case of the ridge topography, the resonant and nonresonant steady states coexist over a wide range of topography heights.
\end{abstract}

\section{Introduction}

The zonal momentum budget of the Southern Ocean is at present not accessible to direct observation. There are many reasons to believe that the main part of the zonal momentum imparted to the fluid by the strong westerly winds around Antarctica is transferred to the solid earth by the action of pressure force on topographic barriers, the so-called bottom form stress (Munk and Palmén 1951), although lateral frictional stresses in the vigorously turbulent boundary current downstream of Drake Passage might also be important. The assumption that bottom form stress plays the major role is supported by the results of an eddy-resolving quasigeostrophic (Marshall et al. 1993) and a diagnostic primitive equation (Stevens and Ivchenko 1997) model of the Southern Ocean.

But the question of identifying the momentum sink is only one part of the puzzle of the Southern Ocean. Other related questions are: How is the strength of the Antarctic Circumpolar Current related to the terms in the momentum balance? If form stress is the main sink of zonal momentum, how is the necessary pressure distribution established, how is the momentum transferred to the

\footnotetext{
* AWI Contribution Number 1455.

+ Current affiliation: Institut für Meereskunde an der Universität Kiel, Kiel, Germany.
}

Corresponding author address: Dr. Christoph Völker, Institut für Meereskunde an der Universität Kiel, Dusternbrooker Weg 20, 24105 Kiel, Germany.

E-mail: cvoelker@ifm.uni-kiel.de depth where this mechanism can work, and what is the role of small-scale turbulence in this process? A discussion of these issues can be found in Olbers (1998).

Eddy-resolving quasigeostrophic models of flow in zonally oriented channels with more or less idealized topography have been a major tool in trying to understand these issues in a highly idealized but at the same time more easily analyzable context (McWilliams et al. 1978, hereafter MHC78; Wolff and Olbers 1989; Treguier and McWilliams 1990; Wolff et al. 1991, hereafter WMO91). Although limited in their physics these models show features, especially the bandlike structure of the eastward current, that resemble the ACC. They also support the concept of Munk and Palmén that bottom form stress is the main sink of zonal momentum.

There have been several attempts to explain the momentum balance in these channel models and with it the strength of the zonal current by simple analytical models. The homogeneous models of Krupitsky and Cane (1994) and of Wang and Huang (1995) are based on the assumption that the topography downstream of Drake Passage prevents all geostrophic contours, that is, contours of constant $f / h$ ( $f$ is the Coriolis parameter and $h$ the total depth of the fluid), from closing around Antarctica. They consider a zonally oriented channel with rigid walls and a bottom topography in the form of a ridge across the channel that is high enough to disrupt all geostrophic contours. Based on a topographic Sverdrup balance in most parts of the flow and an analysis of boundary currents complementing the Sverdrup interior, they find analytical expressions for the strength of the current depending on the topography height. 
Although the explanation of the relative smallness of the zonal current (when compared to a frictionally balanced flow) by the geometry of geostrophic contours in Drake Passage is a very attractive one, it poses some problems. The most obvious is the effect of baroclinicity, which allows a part of the flow to decouple from the $f$ / $h$ contours. Using the information from the FRAM model that the flow is approximately self-similar in the vertical, Krupitsky et al. (1996) have constructed an equivalent barotropic model that resolves this problem but at the price of introducing an unexplained observational element, the equivalent barotropic vertical flow structure, into their model.

A general feature of all these models is the boundary layer structure of the current over the ridge. It consists of an internal boundary current that crosses the channel from the southern to the northern boundary, following roughly the geostrophic contours, that connects two attached boundary currents at these boundaries. These attached currents are indeed equivalent western boundary currents of the Stommel type, as mentioned in Wang and Huang.

The model proposed here differs from the models of Krupitsky and Cane and of Wang and Huang in that it explains the generation of a significant form drag to balance the wind stress by a mechanism, namely, the resonance of baroclinic Rossby waves, that works for isolated topographical obstacles and does not involve any boundary layers. Although we disagree with the statement by Wang and Huang that only the topography around Drake Passage has an essential role in terms of generating form drag, we do not claim that our model is correct and models based on blocked $f / h$ contours are wrong. They both propose mechanisms that could be at work in the balance of the far more complicated Antarctic Circumpolar Current.

The resonance of Rossby waves has been extensively studied to explain the observed multimodality and lowfrequency variability of the atmospheric midlatitude circulation, starting with the work of Egger (1978) and Charney and DeVore (1979). But owing to the much higher average zonal wind speed, compared to the speed of oceanic currents, the main stress has been on the resonance of the faster barotropic waves. There have been generalizations to baroclinic models as well, and formally the model studied in section 2 is similar to the one in Charney and Strauss (1980). Besides different parameter values an important difference is the nature of the forcing (purely baroclinic in the atmospheric case while the forcing is in the top layer for an oceanic model). Other differences will be mentioned in the following. Pedlosky (1981) analyses resonance in an atmospheric two-layer model analytically by an asymptotic expansion instead of low-order truncation. His resonance condition, though, is different from the baroclinic resonance considered here and his focus is on topographic instability and not on the zonal momentum balance.

The paper is organized as follows: In the second sec-
TABLE 1. Fixed parameters of the model.

\begin{tabular}{lll}
\hline \hline & \multicolumn{1}{c}{ Parameter } & \multicolumn{1}{c}{ Value } \\
\hline $\mathrm{Y}$ & Channel width & $1500 \mathrm{~km}$ \\
$\mathrm{H}$ & Channel depth & $5 \mathrm{~km}$ \\
$\epsilon$ & Bottom friction coeff. & $1.1 \times 10^{-7} \mathrm{~s}^{-1}$ \\
$f_{0}$ & Mean coriolis param. & $1.1 \times 10^{-7} \mathrm{~s}^{-1}$ \\
$\beta$ & Gradient coriolis param. & $1.4 \times 10^{-11} \mathrm{~m}^{-1} \mathrm{~s}^{-1}$ \\
\hline
\end{tabular}

tion the resonance mechanism is explained in the completely analytically tractable case of a sinusoidal topography and a highly truncated expansion of the streamfunction fields. After that it is examined whether the resonance mechanism is an artifact of the severe truncation or is found also in models with a higher number of degrees of freedom. The main emphasis is on steady states but a few aspects of time-dependent behavior are also mentioned. Since a sinusoidal topography is a very poor representation of the barrier-like topographies considered in the models of MHC78 and others, the fourth section is devoted to the generalization of the resonance mechanism to more complicated topographies. The last section summarizes the results and draws some conclusions.

\section{The resonance mechanism}

\section{a. Model physics}

The physical setting of the model used in this paper is the same as in the numerical studies of quasigeostrophic channel flow by MHC78 and WMO91 except that subgrid-scale eddies are parameterized in a more simple form. It is a zonally oriented periodic channel of length $X$ and width $Y$ on the $\beta$ plane. The fluid consists of two homogeneous, immiscible layers of slightly different density, the lighter fluid atop the heavier, with layer thicknesses $H_{1}+\xi-\eta$ and $H_{2}+\eta-B$. Here $\xi$ stands for surface and $\eta$ for interface election, $B$ for bottom topography, and $H_{i}$ for undisturbed layer depths. Parameters that are held constant in all following model calculations are listed in Table 1 .

The geostrophic part of the flow in each of the layers is described by a streamfunction $\Psi_{i}$, such that the zonal and meridional velocity fields are $u_{i}=-\partial_{y} \Psi_{i}$ and $v_{i}=$ $\partial_{x} \Psi_{i}$. The time evolution is governed by the equations

$$
\partial_{t} Q_{i}+\mathcal{J}\left(\Psi_{i}, Q_{i}\right)=F_{i}
$$

for the quasigeostrophic potential vorticity (QPV) $Q_{i}$, where the Jacobian $\mathcal{g}(A, B)$ is defined as $\partial_{x} A \partial_{y} B-$ $\partial_{y} A \partial_{x} B$ and the vorticity sources and sinks $F_{i}$ are caused by the action of winds stress, bottom friction, and an interfacial friction that parameterizes the vertical momentum flux (and lateral heat flux) caused by smallscale eddies. The explicit form of the forcing terms is

$$
\begin{aligned}
& F_{1}=\frac{\text { curl } \tau}{H_{1}}-\mu \Delta\left(\Psi_{1}-\Psi_{2}\right), \\
& F_{2}=\mu \Delta\left(\Psi_{1}-\Psi_{2}\right)-\epsilon \Delta \Psi_{2} .
\end{aligned}
$$


curl $\boldsymbol{\tau}$ is the vertical component $\partial_{x} \tau_{y}-\partial_{y} \tau_{x}$ of the rotation of the wind stress. It is assumed that the wind stress is purely zonal with latitude dependence $\tau_{x}=\tau_{0}$ $\sin ^{2}(\pi y / Y)$, which is slightly different from the sinusoidal wind stress profile used in WMO91. Because the spectral model, to be developed below, resolves only scales of motion much greater than the internal Rossby radius $\lambda=\left[g^{\prime} H_{1} H_{2} / f_{0}^{2}\left(H_{1}+H_{2}\right)\right]^{1 / 2}\left(g^{\prime}\right.$ and $f_{0}$ are defined below), the QPV (multiplied by the mean-layer depth $H_{i}$ ) is dominated by the planetary vorticity $f$ (the vertical part of the earth's rotation rate) and a stretching term caused by the topography $B$ and by the elevation of the layer interface height $\eta$

$$
\begin{aligned}
& Q_{1}=\frac{f_{0}}{H_{1}} \eta+f \\
& Q_{2}=\frac{f_{0}}{H_{2}}(B-\eta)+f .
\end{aligned}
$$

The latitude dependence of the Coriolis parameter $f$ is linearized as $f_{0}+\beta y ; \eta$ is related to the streamfunctions by $\eta=\left(f_{0} / g^{\prime}\right)\left(\Psi_{2}-\Psi_{1}\right)$, where $g^{\prime}=g\left(\rho_{2}-\rho_{1}\right) / \rho_{2}$ is the reduced gravity. The stretching vorticity caused by the surface elevation and the relative vorticity $\Delta \Psi$ are neglected in Eqs. (4) and (5). The ratio of relative vorticity to the stretching vorticity is of the order $(\lambda / L)^{2}$ where $L$ is a typical length scale of the flow. The neglect of relative vorticity is one difference to Charney and Strauss (1980) and is responsible for the analytic solvability of the low-order model. Besides the kinematic boundary conditions (periodicity and no mass flow through northern and southern channel boundaries)

$$
\Psi_{i}(x+X, y, t)=\Psi_{i}(x, y, t) \text { and } \partial_{x} \Psi_{\left.i\right|_{y=0, Y}}=0
$$

no further dynamic boundary conditions are needed due to the nonexistence of a term corresponding to a lateral momentum diffusion in the forcing terms (2) and (3).

But in a multiply connected domain such as a channel the QPV dynamics alone are not sufficient to uniquely determine the streamfunction fields: Four auxiliary conditions are needed corresponding to the four time-dependent values of the streamfunctions on the channel walls.

McWilliams (1977) has shown that a consistent set of constraints follows from demanding that the ageostrophic pressure gradient in the momentum balance vanishes in each of the layers when integrated once around an island and from demanding that the layers do not exchange mass. In the channel geometry and with vanishing wind stress on the boundary these conditions can be brought into the simple form

$$
\begin{aligned}
& \left.\oint_{0}^{X} \partial_{y} \Psi_{i}\right|_{y=0} d x=0 \\
& \oint_{0}^{X} \int_{0}^{Y} \eta d y d x=0 .
\end{aligned}
$$

The fourth constraint uses that the addition of a constant value to all streamfunctions does not change the velocity fields. It is

$$
\left.\left(H_{1} \Psi_{1}+H_{2} \Psi_{2}\right)\right|_{y=0}=0 .
$$

Transformation to barotropic and baroclinic streamfunctions $\Phi$ and $\Theta$ is achieved by

$$
\begin{array}{ll}
\Psi_{1}=\Phi+\Theta & \Phi=\left(\alpha \Psi_{1}+\Psi_{2}\right) /(1+\alpha) \\
\Psi_{2}=\Phi-\alpha \Theta & \Theta=\left(\Psi_{1}-\Psi_{2}\right) /(1+\alpha),
\end{array}
$$

where $\alpha=H_{1} / H_{2}$ is the ratio of the mean layer depths. The corresponding barotropic and baroclinic QPV balances will be given in dimensionless form. A timescale $1 /\left|f_{0}\right|$ and a lengthscale $L=Y / \pi$ are defined to scale the dependent and independent variables according to

$t^{\prime}=t\left|f_{0}\right|, \quad\left(x^{\prime} y^{\prime}\right)=\frac{(x, y)}{L}, \quad\left(\Phi^{\prime}, \Theta^{\prime}\right)=\frac{1}{f_{0}^{2} L^{2}}(\Phi, \Theta)$.

The dimensionless width of the channel is $\pi$ and the length is $2 \pi l$, where $l=X / 2 Y$ is a ratio of the channel dimensions. Dimensionless parameters are defined by

$$
\begin{aligned}
& B^{\prime}=B / H, \quad \boldsymbol{\tau}^{\prime}=\boldsymbol{\tau} /\left(f_{0}^{2} L H\right), \quad \beta^{\prime}=L \beta\left|f_{0}\right| \\
& \epsilon^{\prime}=\epsilon /\left|f_{0}\right|, \quad \mu^{\prime}=\mu /\left|f_{0}\right|, \quad \sigma^{\prime}=(\lambda / L)^{2}
\end{aligned}
$$

and the primes are omitted in the following for brevity. From (1) the two QPV balances

$$
\begin{aligned}
& \beta \frac{\partial \Phi}{\partial x}-g(\Phi-\alpha \Theta, B) \\
& =\operatorname{curl} \tau-\frac{\epsilon}{1+\alpha} \Delta(\Phi-\alpha \Theta) \\
& -\frac{1}{\sigma}\left(\frac{\partial \Theta}{\partial t}+\mathcal{g}(\Phi, \Theta)\right)+\beta \frac{\partial}{\partial x}(\Phi+\Theta) \\
& =\frac{1+\alpha}{\alpha} \operatorname{curl} \tau-(1+\alpha) \mu \Delta \Theta
\end{aligned}
$$

can be derived. The barotropic balance (10), which is the average of (1) over the two layers, is diagnostic and linear as a consequence of the neglection of relative vorticity. The sign of the topography term is correct for the Southern Hemisphere where $f_{0}$ is negative. Equation (11) is the QPV balance for the upper layer, expressed in terms of the barotropic and baroclinic streamfunctions. Its nonlinearity is due to the advection of stretching vorticity by the barotropic flow.

\section{b. The simplest low-order model}

The low-order approximation, made in this section, relies on representing the topography by only a few Fourier components. The simplest case is

$$
B(x, y)=b \sin x \sin y
$$

(assuming for simplicity that $l$ and $\alpha$ equal one, although 
some results are given for the general case, too). This suggests to approximate the streamfunction fields also by only a few of the Fourier modes $F_{m, n}(x, y)=\exp (\operatorname{imx})$ $\sin (n y)$, where $m$ is an integer and $n$ a positive integer. Since the streamfunctions do not necessarily vanish on the lateral channel boundaries (this implies no net zonal flow) whereas the Fourier modes do, one has to add a constant and a linear function of latitude in the expansion. It turns out that the simplest model that retains nonlinearity and a feedback of topography on the flow is the one that represents the zonal mean by a (timedependent) constant and the functions ${ }^{1} y$ and $\sin 2 y$ and the deviations of the zonal mean by expix siny and $\exp (-i x) \sin y$. The truncated expansion is thus

$$
\begin{aligned}
\Phi(x, y, t)= & -U(t) y+\phi_{0,2}(t) F_{0,2}(x, y) \\
& +\left(\phi_{1,1}(t) F_{1,1}(x, y)+\text { c.c }\right) \\
\Theta(x, y, t)= & -u(t) y+C(t)+\theta_{0,2}(t) F_{0,2}(x, y) \\
& +\left(\theta_{1,1}(t) F_{1,1}(x, y)+\text { c.c }\right),
\end{aligned}
$$

where c.c. stands for the complex conjugate. There is no spatial constant in the expansion of $\Phi$ because of the constraint (9). Here $U$ and $u$ are the zonal mean barotropic and baroclinic velocities.

The equations of the low-order model are obtained using the orthogonality of the Fourier modes by a projection of the QPV equations, that is, by multiplication of the equation by some $F_{m, n}$, integration over the model domain, and multiplication by a normalization factor. The projection of the barotropic equation (10) yields

$$
\begin{aligned}
0= & -2 \epsilon\left(\phi_{0,2}-\theta_{0,2}\right) \\
& +\frac{b}{2}\left[\left(\phi_{1,1}+\phi_{1,1}^{*}\right)-\left(\theta_{1,1}+\theta_{1,1}^{*}\right)\right]+\tau_{0} \\
0= & -\epsilon\left(\phi_{1,1}-\theta_{1,1}\right)+i \beta \phi_{1,1} \\
& -\frac{b}{2}\left(\phi_{0,2}-\theta_{0,2}+U-u\right) .
\end{aligned}
$$

From the baroclinic equation (11) one obtains

$$
\begin{aligned}
\partial_{t}\left(\theta_{0,2}+u\right)= & -8 \sigma \mu \theta_{0,2}-i\left[\phi_{1,1} \theta_{1,1}^{*}-\phi_{1,1}^{*} \theta_{1,1}\right] \\
& +2 \sigma \tau_{0} \\
\partial_{t} \theta_{1,1}= & -4 \sigma \mu \theta_{1,1}+i \sigma \beta\left(\phi_{1,1}+\theta_{1,1}\right) \\
& -i\left(\left(\phi_{0,2}+U\right) \theta_{1,1}-\left(\theta_{0,2}+u\right) \phi_{1,1}\right) .
\end{aligned}
$$

These are six real equations for nine unknowns. The three missing equations are obtained by insertion of the expansion (12) and (13) into the constraints (7) and (8). This gives

\footnotetext{
${ }^{1}$ A term proportional to siny can be included in the zonal mean, but it is not coupled with the other modes and is quickly damped away.
}

$$
U=2 \phi_{0,2} \quad u=2 \theta_{0,2} \quad C=\frac{\pi}{2} u .
$$

\section{c. Steady states and baroclinic resonance}

Formally the calculation of the steady states of the model is achieved by setting time derivatives to zero and eliminating variables between Eqs. (14) to (18). This leads to a polynomial equation in one of the variables. The resulting polynomial is of third order with coefficients that are complicated functions of the parameters.

Instead of showing this tedious but straightforward procedure we will try to explain the results using the physical meaning of the model equations. Equation (18) shows that the zonal-mean fields are completely specified by $U$ and $u$ alone. The complex wave amplitudes $\phi_{1,1}$ and $\theta_{1,1}$ may be expressed in terms of a (real) amplitude and a phase shift relative to the topography:

$$
\phi_{1,1}=P e^{-i(\varphi+\pi / 2)} \quad \theta_{1,1}=T e^{-i(\vartheta+\pi / 2)} .
$$

The phases $\varphi$ and $\vartheta$ are defined such that a positive value corresponds to a westward shift of the streamfunction maxima, relative to the location of the topography maximum. Inserting this into (14) and (16) and using the relations (18) to eliminate $\phi_{0,2}$ and $\theta_{0,2}$ one obtains

$$
\begin{aligned}
& 0=\tau_{0}-\epsilon(U-u)-b(P \sin \varphi-T \sin \vartheta), \\
& 0=\tau_{0}-2 \mu u-\frac{1}{\sigma} P T \sin (\varphi-\vartheta) .
\end{aligned}
$$

Although these equations are derived by projection of the vorticity Eq. (10) and (11), they can easily be identified as statements on the zonal momentum balance: Eq. (19) states that the input of zonal momentum by $\tau$ must be balanced either by bottom friction, proportional to the bottom velocity $U-u$ or by bottom form stress, which depends on the amplitude and phase shift of a standing wave in the bottom pressure. Equation (20) states that in a stationary state the zonal momentum can leave the upper layer only by interfacial friction (proportional to the vertical shear of zonal velocity $u$ ) or by interfacial form stress, that is, by the action of an anomaly of the pressure in the first layer, which works against an elevation of the interface. This gives a term that depends on the product of the amplitudes $P T$ and on the phase difference $\varphi-\vartheta$.

Equation (17) can be used to express the baroclinic wave amplitude $T$ and the phase difference $\varphi-\vartheta$ in terms of the barotropic amplitude $P$ and the zonal velocities. One obtains after some algebra

$$
\begin{aligned}
& T \sin (\varphi-\vartheta)=P \frac{4 \sigma \mu(\tilde{u}+\sigma \beta)}{(4 \sigma \mu)^{2}+(\tilde{U}-\sigma \beta)^{2}} \\
& T \cos (\varphi-\vartheta)=P \frac{(\tilde{U}-\sigma \beta)(\tilde{u}+\sigma \beta)}{(4 \sigma \mu)^{2}+(\tilde{U}-\sigma \beta)^{2}},
\end{aligned}
$$




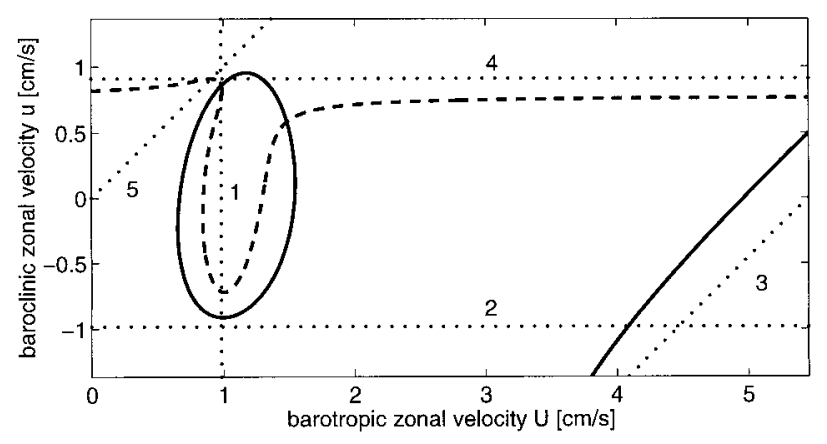

FIG. 1. The curves determined by the barotropic (solid line) and baroclinic (dashed line) momentum balances in $U, u$ space. Parameter values are $\tau=3 \times 10^{-5} \mathrm{~m}^{2} \mathrm{~s}^{-2}, \mu=3 \epsilon, g^{\prime}=0.01 \mathrm{~m} \mathrm{~s}^{-2}$, and $b$ $=100 \mathrm{~m}$. The dotted lines are explained in the text.

where the abbreviations $\tilde{U}=U+\phi_{02}=3 U / 2$ and $\tilde{u}$ $=u+\theta_{02}=3 u / 2$ have been introduced. Resonance, that is, a high wave amplitude $T$ and a high value of the interfacial form stress, proportional to (21), occur when $\tilde{U}$ is near $\sigma \beta$, which is the baroclinic Rossby wave speed, that is, when the excited Rossby waves are trapped near the topography by the counteraction of wave propagation and advection. It is interesting to note that the first expression (and thus the interfacial form stress) depends linearly on the interfacial friction parameter, in accordance with the Eliassen-Palm theorem, which states in this framework that the feedback of waves on the mean flow can happen only via frictional processes.

For the barotropic wave component one obtains an even more complicated expression using (15), but in contrast to the baroclinic wave component there is no resonance when $\tilde{U}$ equals the baroclinic Rossby wave speed:

$$
\begin{aligned}
P \sin \varphi & =\frac{b(\tilde{U}-\tilde{u})}{2} \frac{R}{R^{2}+S^{2}} \\
P \cos \varphi & =\frac{b(\tilde{U}-\tilde{u})}{2} \frac{S}{R^{2}+S^{2}},
\end{aligned}
$$

$R$ and $S$ are abbreviations for

$$
\begin{aligned}
& R=\epsilon-\epsilon \frac{(\tilde{U}-\sigma \beta)(\tilde{u}+\sigma \beta)}{(4 \sigma \mu)^{2}+(\tilde{U}-\sigma \beta)^{2}} \\
& S=\beta-\epsilon \frac{4 \sigma \mu(\tilde{u}+\sigma \beta)}{(4 \sigma \mu)^{2}+(\tilde{U}-\sigma \beta)^{2}} .
\end{aligned}
$$

Expressing now the wave amplitudes and phases as functions of the mean zonal velocities $U$ and $u$, the momentum equations (19) and (20) become implicit equations in the mean zonal velocities that define curves in the $(U, u)$ plane. Intersections of these two curves are steady states of the model.

Figure 1 displays these curves. A weak wind stress forcing $\left(\tau=3 \times 10^{-5} \mathrm{~m}^{2} \mathrm{~s}^{-2}\right)$ was chosen here to make the structure more obvious; at $\tau=10^{-4} \mathrm{~m}^{2} \mathrm{~s}^{-2}$, which is used in the rest of the paper and is probably nearer to ACC conditions, the solution curve of the baroclinic momentum equation becomes slightly more complicated.

To aid the interpretation five auxiliary lines (labeled 1 to 5) are included in the plot. The first line shows the location of the resonance $\tilde{U}-\sigma \beta=0$. The second is defined by the vanishing of $\tilde{u}+\sigma \beta$; below this line the interfacial form stress is a source of eastward momentum. The third and fourth are the solution curves of the barotropic and baroclinic momentum balances for vanishing topography, $\tau-\epsilon(U-u)=0$ and $\tau-2 \mu u=$ 0 . On the fifth line, $U-u=0$, the bottom zonal velocity and both wave amplitudes vanish.

The solution curve for the baroclinic momentum equation (20) runs near line 4, where the vertical shear is big enough to balance the wind stress mainly by interfacial friction. Only for $U$ near resonance (line 1) a strong baroclinic wave amplitude leads to a strong vertical momentum exchange by interfacial form stress, and balance (20) can be fulfilled with low (even negative) values of $u$.

The solution curve for the barotropic momentum equation (19) consists of two disjoint curves; the first is close to the frictionally controlled solution without topography (line 3 ), where the bottom velocity $U-u$ is high enough to balance the wind stress. The second, closed curve near the resonance (line 1) is caused by the contribution of a high baroclinic wave amplitude to the bottom form stress.

The two curves intersect in three points, corresponding to three steady states, of which two are near resonance and the third is near a frictionally balanced flow.

When changing the height of the topography, the number and location of steady states varies in a characteristic way. Figure 2 shows the barotropic and baroclinic mean zonal velocities as a function of topographic amplitude. For vanishing topography the flow is purely zonal; frictional processes balance the zonal momentum input. With increasing topography height, the zonal velocities diminish and form stress becomes more and more important, but the flow is still definitely superresonant. At a critical topographic amplitude (for our parameter set near $380 \mathrm{~m}$ ) two new, resonant steady states appear in a saddle-node bifurcation. They are characterized by high amplitudes of a stationary baroclinic wave and a barotropic zonal velocity near resonance, but they differ in the phase shift between the barotropic and baroclinic wave. The number of steady states decreases again, when one of the resonant and the superresonant state join and vanish in a second saddlenode bifurcation. The remaining resonant steady state tends asymptotically against a state where $U$ and $u$ are equal and $u=\tau / 2 \mu$ (this is the intersection of lines 4 and 5 in Fig. 1). In this asymptotic state the mean zonal velocity in the lower layer and thus bottom friction vanishes, so the barotropic momentum balance requires a high bottom form stress. On the other hand, the inter- 

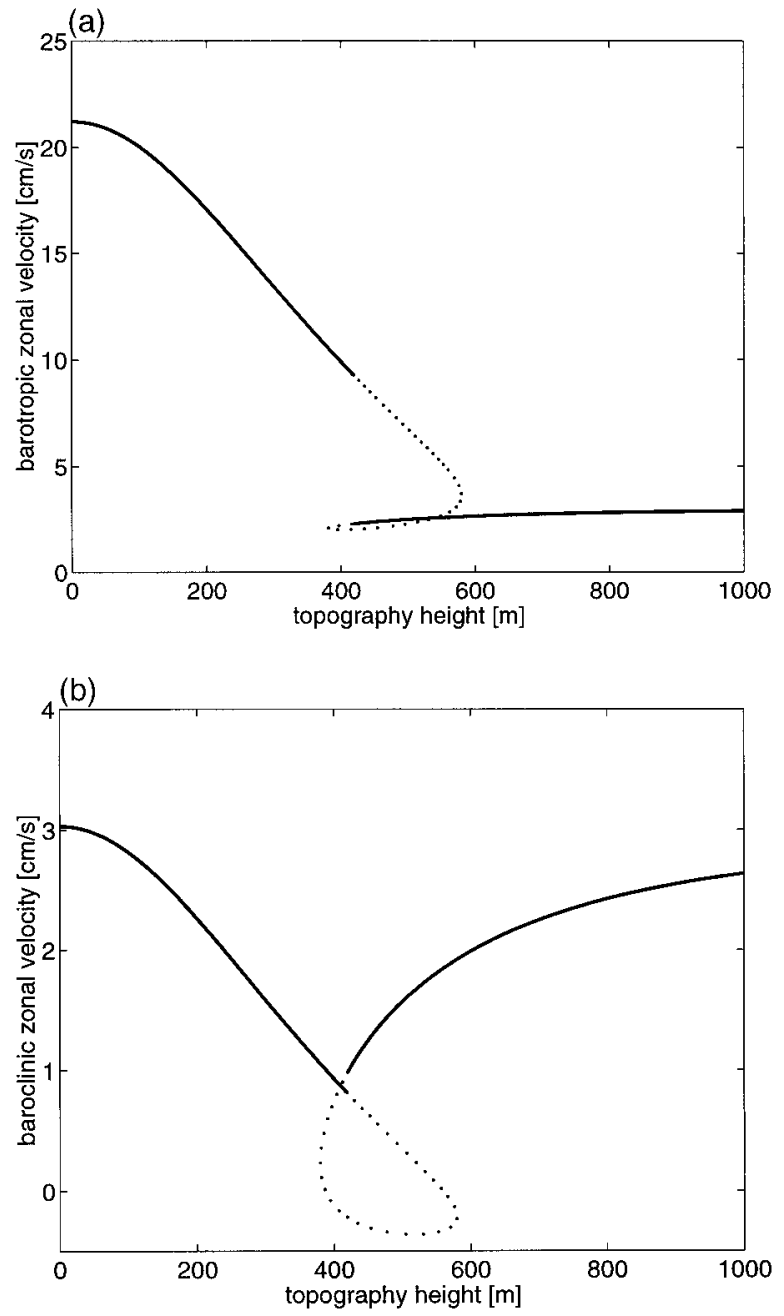

FIG. 2. Barotropic and baroclinic zonal velocities of the steady solution of the low-order equations with varying topographic amplitude. Unstable steady states are indicated by a dotted line. Parameter values are identical to the values in Fig. 1 except $\tau=10^{-4} \mathrm{~m}^{2}$ $\mathrm{s}^{-2}$.

facial form stress vanishes, caused by a vanishing phase difference between the barotropic and baroclinic waves; the baroclinic momentum input is balanced by interfacial friction.

The superresonant and the resonant steady states have characteristically different streamfunction patterns. For the parameter values used in the calculation of Fig. 2, the model possesses three steady states at a topographic height of $400 \mathrm{~m}$, of which only the superresonant one is stable. Figure 3 compares these steady states and Table 2 shows their transports and integral momentum balances.

The superresonant state is characterized by a strong eastward current in both layers that is deflected northward over the topographic elevation and southward over the depression. There are two large closed circulation cells, one cyclonic over the elevation and one anticy- clonic over the depression. The whole pattern resembles the form of the geostrophic contours $\beta y+f_{0} B /\left(H_{1}+\right.$ $H_{2}$ ) = const, as would be expected for this relatively barotropic current. The phase shift of the current, relative to the topography, that is responsible for the form drag in its barotropic and baroclinic momentum balances is too small to be recognized clearly in the figure. The two other steady states have very similar barotropic mean zonal velocities near resonance, but differ strongly in most other respects. The second state, which represents the transition from the superresonant states to the resonant ones, has deflections from the zonal path that are stronger in the upper layer and weaker in the lower layer than in the superresonant state. The location of the closed circulation cells is about the same. The whole current is much weaker (note the different contour interval in the pictures in Fig. 3). The main difference of the third, resonant steady state to the other two states is the direction of the deflection of the zonal current in the lower layer: It is poleward over the topographic elevation and equatorward over the depression. This is a typical feature of all the resonant steady states for higher topographic amplitudes.

In Olbers and Völker (1996) the steady states of this low-order model are compared to time-mean states of an eddy-resolving numerical channel model with sinusoidal topography but without an explicit interfacial friction. Figure 4 shows the time-mean streamfunction fields for a numerical experiment with a channel length to width ratio of $4000 \mathrm{~km} / 1500 \mathrm{~km}(l=4 / 3)$ and a topography height of $500 \mathrm{~m}$. The pattern is very similar to a stable superresonant state of the low-order model, setting $\mu=2.9 \times 10^{-7} \mathrm{~s}^{-1}$. A comparison of transports and momentum balances gives reasonable correspondence, given the simplicity of the low-order model: The zonal mass transports are 404 and $239 \mathrm{~Sv}\left(\mathrm{~Sv} \equiv 10^{6}\right.$ $\mathrm{m}^{3} \mathrm{~s}^{-1}$ ) in the two layers of the numerical model, and 446 and $363 \mathrm{~Sv}$ in the low-order model. In the numerical model $74 \%$ of the momentum transfer from the upper to the lower layer is caused by form stress by the stationary pressure anomalies, compared to $72 \%$ in the low-order model. Bottom form stress accounts for $71 \%$ of the total momentum loss to the earth in the numerical case and $54 \%$ in the low-order model. For higher topographies the correspondence decreases, probably due to the inaedequacy of the interfacial friction parameterization.

The low-order model described here is formally quite similar to the baroclinic atmospheric model by Charney and Strauss (1980, hereafter CS). This necessitates a description of the differences both to the barotropic resonance mechanism, decribed by Charney and DeVore (1979, hereafter CDV), and the baroclinic model by CS.

The location of resonance in barotropic models like CDV does depend on the length scale of the topography, while it does not in the baroclinic case. This is due to the nondispersive nature of long baroclinic Rossby waves. We thus do not expect a broadening of the res- 

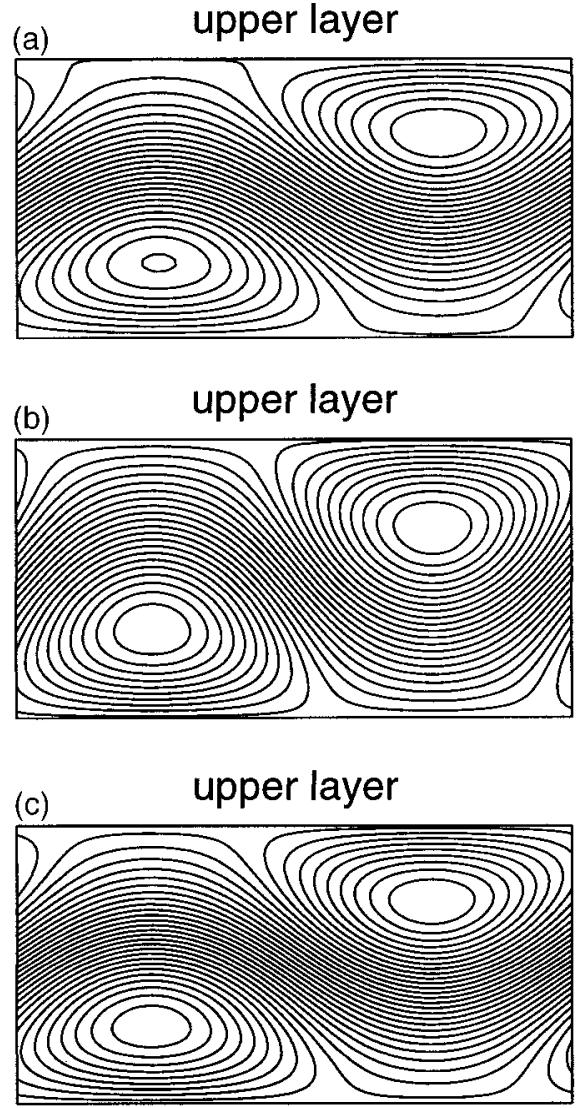

lower layer

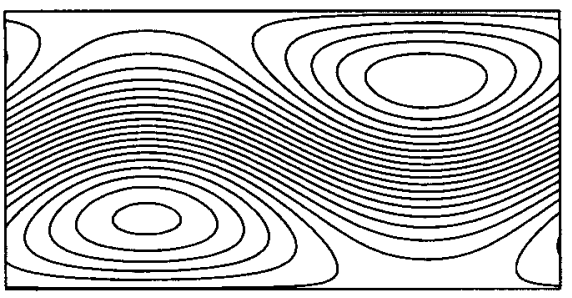

lower layer

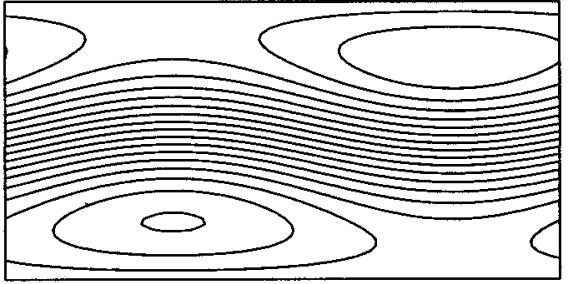

lower layer

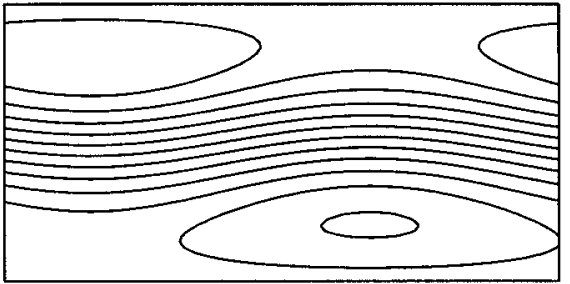

FIG. 3. Isolines of the streamfunction for the three steady states of the low-order model at a topography height of $400 \mathrm{~m}$. The ordering of the states is the same as in Table 2; i.e., the upper two panels show the streamfunction in both layers for the superresonant state, the middle pictures for the transitional resonant state, and the lower panels for the stable resonant state. The contour interval is $10000 \mathrm{~m}^{2} \mathrm{~s}^{-1}$ for the upper panels and $2500 \mathrm{~m}^{2} \mathrm{~s}^{-1}$ for the middle and lower panels.

onance condition for realistic topographies containing many Fourier modes. In the barotropic case this broadening can destroy the existence of multiple steady states (Tung and Rosenthal 1985). A second difference to CDV is the existence of an oscillatory instability that can destabilize the superresonant and the resonant steady state. The instability, also found in CS, occurs for topographic heights not too far away from the transition to the unstable resonant state; there is even a small

TABLE 2. Mass transports and relative size of terms in the zonal momentum balance for the three steady states of the low-order model for $b=400 \mathrm{~m}$, shown in Fig. 3. The transports are given in Sverdrups for each layer. The terms in the momentum balances are interfacial form stress (IS), interfacial friction (IF), bottom form stress (BS), and bottom friction (BF), and are given in percent of the momentum input by the wind stress. IF + IS and BF + BS total $100 \%$.

\begin{tabular}{crrrrrrr}
\hline \hline & \multicolumn{2}{c}{ Transports (Sv) } & & \multicolumn{4}{c}{ Momentum balances (\%) } \\
\cline { 2 - 3 } \cline { 5 - 8 } State & $T_{1}$ & $T_{2}$ & & IS & IF & BS & BF \\
\hline 1 & 405 & 336 & & 70 & 30 & 51 & 49 \\
2 & 71 & 79 & & 103 & -3 & 88 & 12 \\
3 & 112 & 54 & & 75 & 25 & 92 & 8 \\
\hline
\end{tabular}

region in parameter space where none of the three steady states of the model is stable. Here complicated timedependent behavior is found that is linked to growth, propagation, and destruction of a baroclinic Rossby wave. This is described in more detail in Völker (1996).

The main difference of the present model from that of CS is the nature of the forcing; this seemingly small difference however leads to strong differences in the model steady states. The purely baroclinic forcing in CS allows for a Hadley cell solution, where the frictionally balanced flow is confined to the upper model layer and does not interact with topography. Charney and Strauss find up to four additional solutions that exist only over a limited range of forcing strengths. Two of these solution branches have negative, that is, westward, flows in the lower layer, which never happens in the model presented here, and two have barotropic zonal velocities higher than the frictionally balanced flow, indicating that the interaction with topography acts as a source of eastward barotropic momentum. The zonal momentum balances in CS are thus quite different from the ones in the model presented here. Another difference 


\section{layer 1 , time mean}

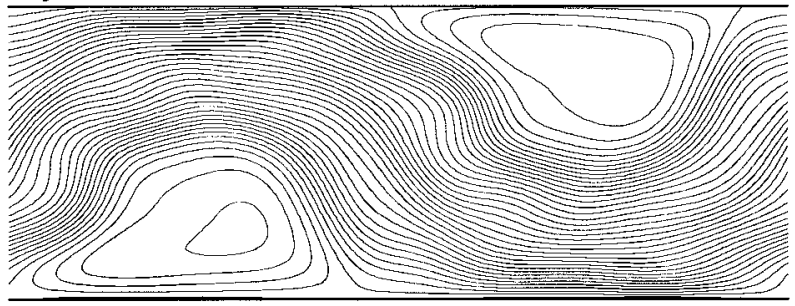

\section{layer 2}

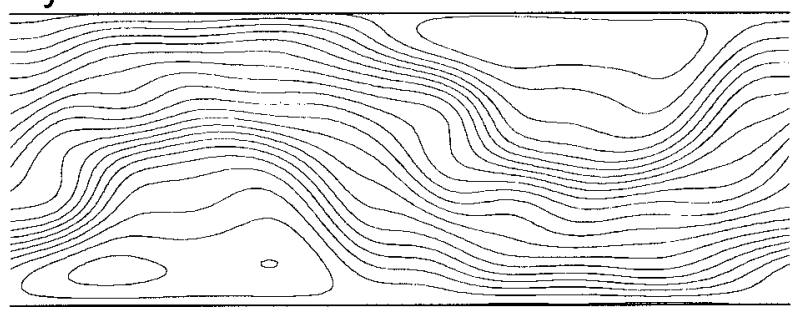

FIG. 4. Isolines of the time-mean streamfunction from an eddyresolving numerical model experiment with sinusoidal topography and two fluid layers of equal thickness. The channel length is 4000 $\mathrm{km}$, the topography height $500 \mathrm{~m}$, the reduced gravity $0.01 \mathrm{~m} \mathrm{~s}^{-2}$, and the biharmonic friction coefficient $10^{10} \mathrm{~m}^{4} \mathrm{~s}^{-1}$. All other parameters are the same as in Table 1. Isoline spacing is $5000 \mathrm{~m}^{2} \mathrm{~s}^{-1}$.

is that the internal Rossby radius in CS is of the same order of magnitude as the wavelength of the low-order modes, meaning that barotropic and baroclinic Rossby waves have similar phase speeds. This makes it difficult to discriminate between barotropic and baroclinic resonance. The neglection of relative vorticity in the present model is not responsible for the differences to CS: Inclusion of relative vorticity in the low-order equations and their numerical solution leads to steady states that differ only slightly from the ones presented.

\section{d. Other parameter dependences}

Although a full discussion of dependence of the model outcomes on wind stress, stratification, and other parameters is not intended here, some remarks may help explain the resonance mechanism. For a more complete discussion see Völker (1996).

The existence of a resonant steady state obviously depends on the sign of the wind stress forcing: For westward forcing the propagation of topographically excited Rossby waves is in the same direction as the advection by the wind-driven circulation. We thus do not expect a resonance to occur, and this is indeed what is found in the model. The mean barotropic zonal velocity in the absence of topography is directly proportional to the strength of the wind stress; for resonance to be possible this velocity not only has to be opposed but must also be larger than the (westward) propagation velocity of baroclinic Rossby waves.

Since interfacial friction is only a parameterization of vertical momentum transport by eddies, it is reassuring that the value of the interfacial friction parameter $\mu$ has no qualitative influence on the existence of resonant and nonresonant model states: A change in $\mu$ merely changes the location of the topography interval where the transition from a friction-dominated solution to a resonant solution happens and where three steady states coexist. For $\mu / \epsilon=25$ this coexistence interval is between 210 and $310 \mathrm{~m}$; for $\mu / \epsilon=0.5$ it is between 1010 and $1160 \mathrm{~m}$. Variations in the height ratio of the model layers $\alpha$ and in the ratio of the channel dimensions $l$ have a similar effect: A decrease in $\alpha$ or an increase in $l$ leads to a shift of the coexistence region to greater topographic heights.

The influence of stratification can be understood from a scaling invariance. A variation in $\sigma$ is equivalent to a variation in the strength of the wind stress forcing: If $\Psi(t, x, y), \Theta(t, x, y)$ is a solution of the model equations for parameter values $\tau_{0}, \sigma$, then $a \Psi(a t, x, y), a \Theta(a t, x, y)$ is a solution for parameters $a \tau_{0}$ and $a \sigma$. Physically this invariance expresses that it is the ratio between winddriven flow velocity and Rossby wave speed that determines the resonance behavior.

\section{Resonance in a moderate-order model}

The model considered so far has the advantage of being completely analytically tractable, but through its severe truncation it excludes some important nonlinear physical processes. Its probably most drastic simplification is the absence of any net meridional flux of zonal momentum by Reynolds stresses.

One might therefore ask whether the resonance mechanism and the multiple steady states it produces are merely artifacts of the truncation. In a barotropic channel model with a more complicated topography it has been demonstrated by Tung and Rosenthal (1985) that the nonlinear interaction of a large number of Fourier modes tends to counteract the resonance and to destroy the existence of multiple steady states.

This question is investigated by looking at the steady states and the dynamics of a less severely truncated spectral model, which still is far from the complexity of the eddy-resolving numerical models by MHC78 and WM091. The model differs from the low-order model only in the spectral representation of the streamfunction fields. The expansion includes all Fourier modes with wavenumbers in the triangle defined by $|m|+n \leq M$ +1 and $n>0$. For $M=1$ the low-order model discussed above is obtained. Model equations are again derived by projecting the barotropic and baroclinic vorticity equations (10) and (11) on the Fourier modes and by inserting the expansion into the auxiliary conditions (7) and (8). This is deferred here to an appendix. Results 

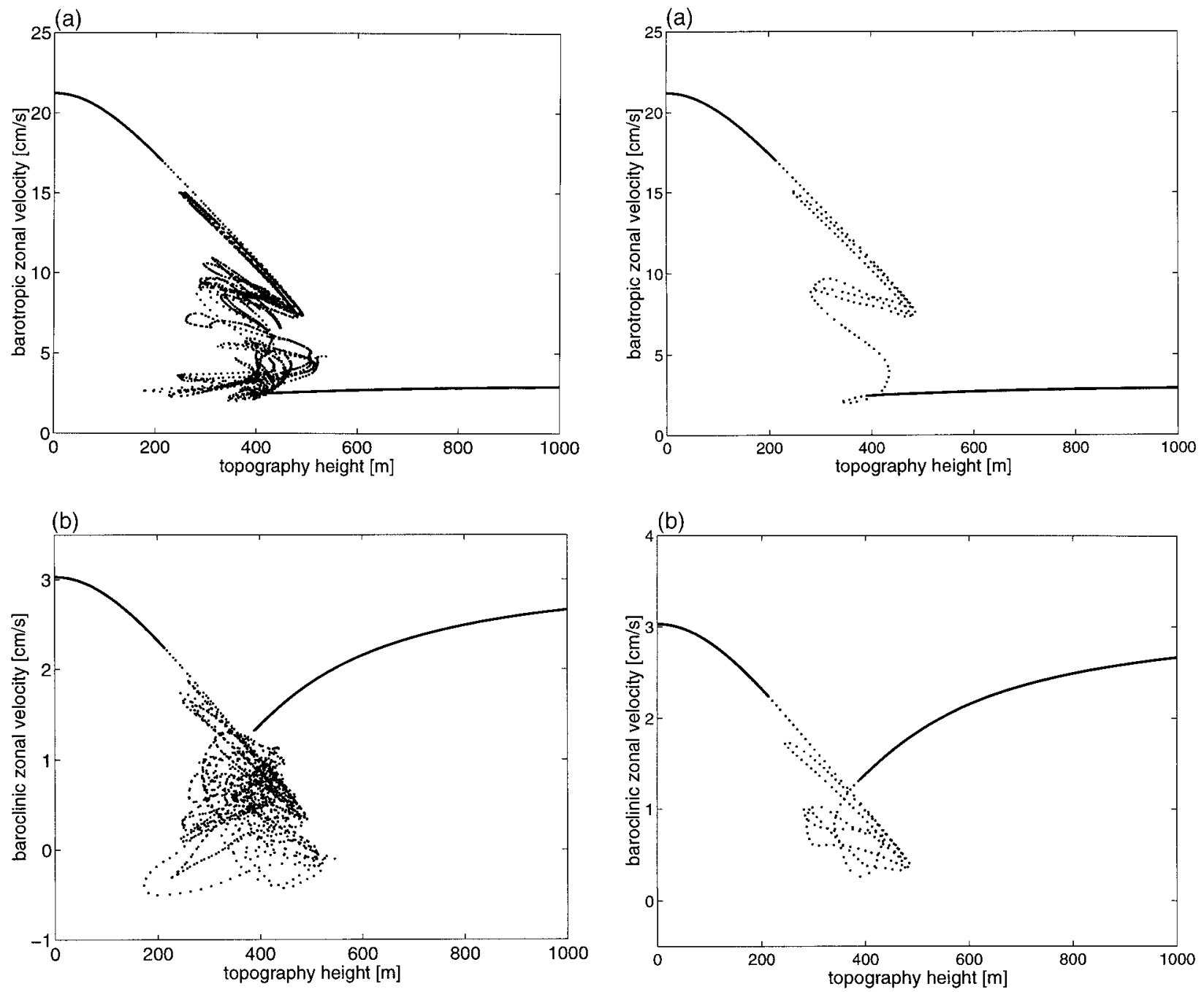

FIG. 5. Barotropic and baroclinic zonal velocities of the steady states of the moderate-order model for varying topographic amplitude. The other parameter values are identical to the values used in Fig. 2. Unstable steady states are indicated by a dotted line.

are shown for a model with $M=5$, leading to a total of $2(M+1)^{2}+3=75$ degrees of freedom.

The steady states of this model were calculated by means of a continuation algorithm, following the location of the steady states when a parameter (in our case topography height) is continuously changed. Keeping track of the derivative of the model equations with respect to the model variables and the changed parameter allows a detection of bifurcations where new branches of solutions appear. For a detailed decription of the method the reader is referred to Parker and Chua (1989). A self-developed numerical algorithm was used for continuation and detection of bifurcations; results were checked using the bifurcation analysis tool AUTO by Doedel and Wang (1995).

Using this method and stating from the known so-

FIG. 6. As in the previous figure but showing only the zonal velocities of the main solution branch.

lution for vanishing topography, steady states of the model equations and their stability properties were obtained in the parameter range from 0 to $1000 \mathrm{~m}$ topographic height. Figure 5 displays the mean zonal velocities obtained in this way. The graph should be compared to Fig. 2, which shows the same variables for the low-order model.

The most prominent difference to the low-order model is the number of steady states existing in the intermediate parameter range. The graph consists of a main solution branch that extends over the whole parameter range and a number of side branches that exist only over a limited range of topographic heights. The side branches bifurcate in pairs from the main branch and end on it in the same way.

The zonal velocities of the main branch, displayed separately in Fig. 6, are for high and low topographic amplitudes almost identical to those on the solution 
curve of the low-order system, meaning that a frictionally balanced and a resonantly balanced state exists in both models. The transition range with several steady states is shifted to somewhat lower topographic values in the moderate-order model and the number of steady states is greater. In this parameter range there are some steady states with a zonal barotropic velocity between the extremes of a resonantly and a frictionally controlled state. In contrast to the low-order model there is now a relatively large parameter range where no stable steady state exists. The solutions on the main branch have the symmetry $\phi_{m, n}=\theta_{m, n}=0$ for $m+n$ odd. This symmetry, however, does not imply the vanishing of net meridional momentum transport by Reynolds stress terms. The amplitudes of the Fourier modes with wavenumbers higher than $(1,1)$ are about one order of magnitude smaller than the amplitude of the $(1,1)$ mode and have their maximum values in the topographic range where the transition from a frictionally to a resonantly controlled state occurs, indicating a higher degree of nonlinearity there.

The number and location of the secondary solution branches is somewhat dependent on the truncation of the spectral model; for $M=5$ there are eight bifurcation points on the main branch where a pair of secondary solution branches originates. These branches represent symmetry-breaking solutions of the model equations with nonvanishing Fourier amplitudes for $m+n$ odd and more complicated nonlinear balances. Nevertheless, their zonal velocities are never very different from values on the main branch, so the momentum balances do not seem to be much affected. The secondary branches all close on themselves, forming a loop that in one case intersects the main branch several times. The symmetrybreaking solutions exist only near the parameter range where the main branch has multiple steady states, that is, where the topography is high enough to allow for the existence of resonant states, but not too high as to exclude all other possible balances. All steady states on the secondary solution branches are unstable, except in a small topographic height interval between $426 \mathrm{~m}$ and $436 \mathrm{~m}$, where one steady state on a secondary solution branch becomes stable.

Although there are now nine steady states for a topographic height of $400 \mathrm{~m}$ on the main branch alone, most of these states differ mainly in the width and strength of the jet that passes through the channel from the superresonant state of the low-order model (shown in Fig. 3). The overall structure of the fields with a northward deflection over topographic elevation in both layers agrees well. Only the stable, resonant steady state differs; not surprisingly it resembles the resonant steady state (Fig. 3, lower picture) of the low-order model, with a southward deflection of the weak current over topographic elevation in the lower layer.

Some numerical integrations of the model equations were performed to obtain an impression of the timedependent behavior of the model. For a topographic height of $200 \mathrm{~m}$, attraction toward the only steady state was found, but, given the high dimension of the phase space, a few integrations are of course not sufficient to rule out the existence of periodic or even chaotic orbits. The same holds for a topographic height of $600 \mathrm{~m}$. For a height of $300 \mathrm{~m}$, where no stable steady states exist, complicated, perhaps chaotic, behavior was found.

\section{Resonance over more complex topography}

A single Fourier mode is a very poor representation even of the simple and smooth topographic obstacles that have been used in numerical studies of quasigeostrophic channel flow, not to speak of realistic topographies. A topography that has been widely used in investigations of the dynamics of the Antarctic Circumpolar Current with numerical quasigeostrophic channel models (MHC78; Wolff and Olbers 1989; WMO91) is an isolated Gaussian ridge, $B(x, y)=B_{0} b_{1}(x) b_{2}(y)$, with

$$
\begin{aligned}
& b_{1}(x)=e^{-\left(x-x_{0}\right)^{2} / 2 s^{2}} \\
& b_{2}(y)= \begin{cases}e^{-\left(y-y_{s}\right)^{2} / 2 s^{2}} & \text { for } y>y_{s} \\
1 & \text { for } y_{s} \geq y>y_{n} \\
e^{-\left(y-y_{n}\right)^{2} / 2 s^{2}} & \text { for } y_{n} \geq y,\end{cases}
\end{aligned}
$$

where $s$ defines the width of the ridge and $y_{s}$ and $y_{n}$ its meridional extent. In the following it is studied whether resonance of baroclinic Rossby waves also occurs in flow over such a topography and whether this resonance leads to the existence of multiple steady states. The width and location of the Gaussian ridge are set to $s=$ $150 \mathrm{~km}, y_{s}=500 \mathrm{~km}$, and $y_{n}=1000 \mathrm{~km}$ such that it blocks about one-third of the channel width. The Fourier expansion of the streamfunction and of the topography are truncated using a triangular truncation with maximal zonal wavenumber $M=5$ as in the previous section. Figure 7 shows the form of the truncated topography.

A priori we would expect resonance to occur because of the dispersionless nature of baroclinic Rossby waves in the long-wave limit used in our model calculations. Although the interaction of the wind-driven zonal flow with the topography now excites many different Fourier modes, the resonance condition for all these modes is identical. But still the dependence on parameter values, such as topographic height, might be completely different, and the higher degree of nonlinearity, implied by the topographic excitation of many harmonics, might change the simple resonance picture considerably.

To separate the effect of interaction with topography from the effect of increased nonlinearity two different models are studied. The first one is a quasilinear model in the sense of Davey (1980). Discriminating between the zonal mean of the streamfunctions and the deviations thereof (the "wave field") it neglects the feedback of wave-wave nonlinear terms and of wave-topography interaction on the wave field. Formally this is achieved by neglecting terms in the equation for the $(m, n)$ mode 


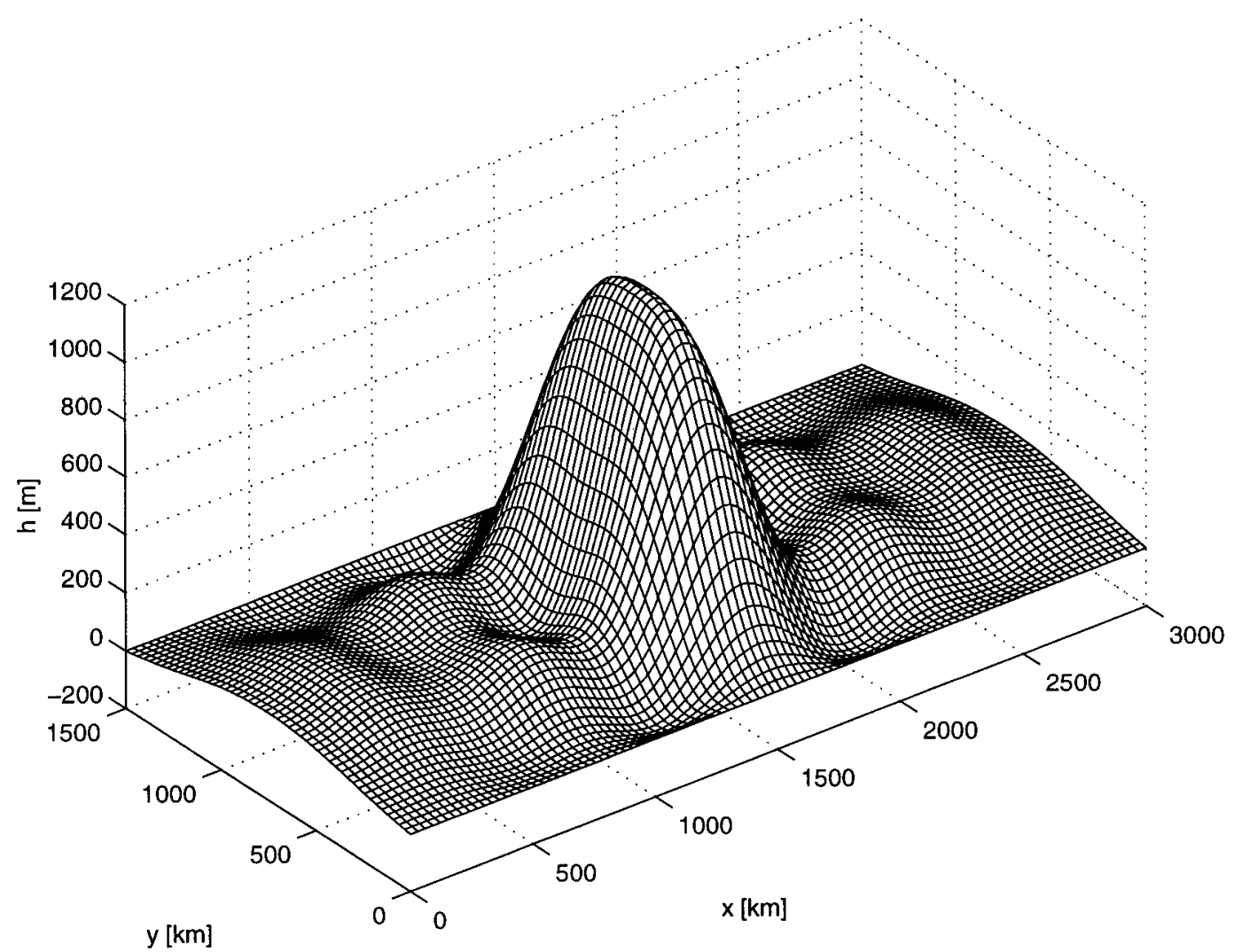

FIG. 7. The truncated version of the Gaussian topography, using a triangular truncation in wavenumber space with maximal zonal wavenumber $M=5$. The rms difference to the exact Gaussian topography is below $10 \%$ and the maximum difference is slightly larger than $20 \%$.

that are products of amplitudes of $\left(m^{\prime}, n^{\prime}\right)$ and $\left(m^{\prime \prime}, n^{\prime \prime}\right)$ modes of streamfunction or topography, if none of $m$, $m^{\prime}$ and $m^{\prime \prime}$ is zero.

The second model contains the complete nonlinearities and is identical to the moderate-order model of the last section, except that it contains interactions with a higher number of topographic modes.

As before, the steady states of these models are found by following their location as the topography height is increased. For the quasilinear model the zonal velocities resulting from this calculation are shown in Fig. 8. Except in the range between 500 and $700 \mathrm{~m}$ the topographic height curves agree quite well qualitatively with the corresponding ones from the low-order model with sinusoidal topography (Fig. 2). They show the same transition from a stable superresonant steady state where the momentum input is balanced mainly by interfacial and bottom friction to a resonant steady state where baroclinic resonance leads to a high bottom form drag. In part of the transitional region there are more than three steady states, but there are no side branches bifurcating from the main branch of solutions, as in the moderate-order model with sinusoidal topography. The absence of side branches is explained by the lack of symmetry of the main solution branch: The interaction of the mean zonal flow with a Gaussian topography excites all Fourier modes directly, so there is no symmetry (as for sinusoidal topography) $\phi_{m, n}=\theta_{m, n}=0$ for $m+n$ odd that can be broken. Although there is the qualitative agreement in the topographic dependence of the mean zonal velocities, we must not forget that the streamfunction patterns belonging to these velocities reflect the structure of the topography and thus are fundamentally different from those in the low-order model.

Passing now to the fully nonlinear model (Fig. 9), some new features can be noted that are observed neither in the nonlinear model with sinusoidal topography nor in the quasilinear model. There is no continuous transition from a superresonant state to a resonant one as the topographic height is increased, at least not in the parameter range that was investigated. Instead, the model possesses one superresonant state with high zonal velocities over the whole topographic range; there are no bifurcations from this solution branch. The additional feedback on the wave part of the streamfunctions, compared to the quasilinear model, seems to stabilize the high-index state and to prevent the buildup of a strong form drag. This seems to agree with the result of Tung and Rosenthal (1985), that in a barotropic moderateorder model with "realistic" topography multiple steady 

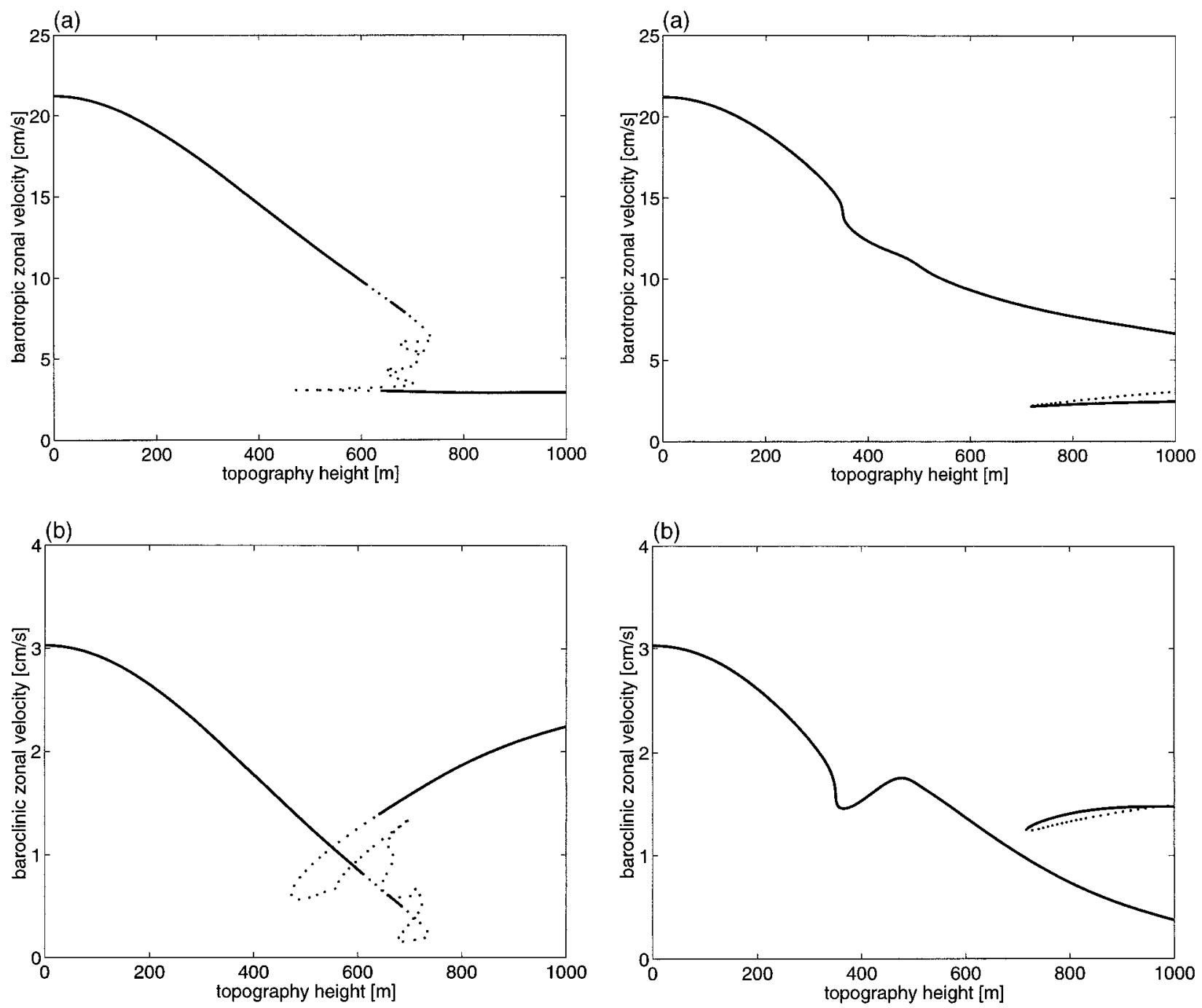

FIG. 8. Zonal velocities in the quasilinear model of steady channel flow over Gaussian topography as a function of the obstacle height.

states disappear, but which are present in low-order models.

In contrast to Tung and Rosenthal one finds two additional resonant solutions for higher topographies, one of which is stable over a wide ranges of topography. They can easily be recognized by their barotropic zonal velocity near the resonant value of somewhat less than $3 \mathrm{~cm} \mathrm{~s}^{-1}$. These resonant branches of steady states would not have been found by the bifurcation routine alone because there is no direct connection with the main solution branch in the parameter range considered here. They were found by taking the single, resonant steady state of the quasilinear model for 1000-m topographic height as the initial condition in a numerical integration of the time-dependent fully nonlinear model. This integration ended on a resonant steady state of the nonlinear model. This new steady state was then taken as a starting point for the bifurcation routine, going

FIG. 9. Zonal velocities in a fully nonlinear model of steady channel flow over Gaussian topography as a function of the obstacle height.

backward toward lower values of topography height. There might be a continuous transition from the superresonant state to the resonant ones for significantly higher topography that, however, violates quasigeostrophic scaling.

The main new feature in Fig. 9 is the existence of two stable steady states, one blocked and one unblocked, over a wide range of topographic heights. This might suggest that the explanation of the observed low-frequency variability in numerical eddy-resolving channel models (MHC 1978; WMO 1991) is indeed the existence of several steady states of the large-scale circulation, between which the system is driven by the action of small-scale turbulence. This explanation has been proposed in Treguier and McWilliams (1990), where the authors refer to the barotropic model of Charney and DeVore (1979). 

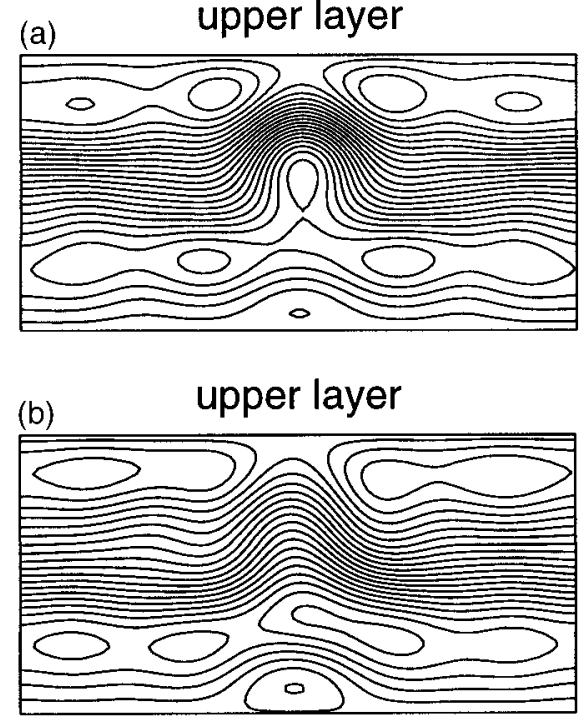

(c)

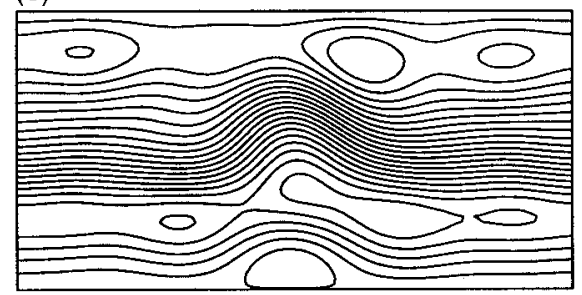

lower layer

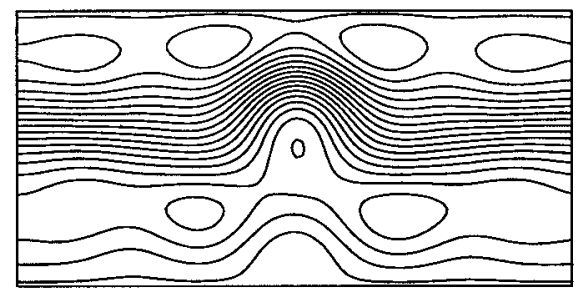

lower layer

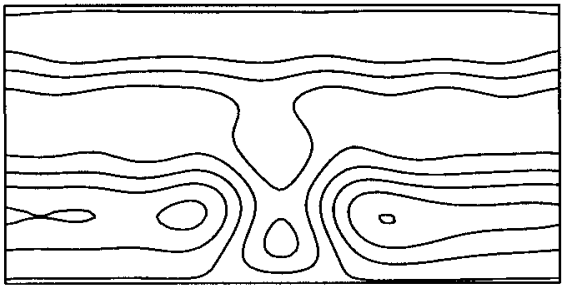

lower layer

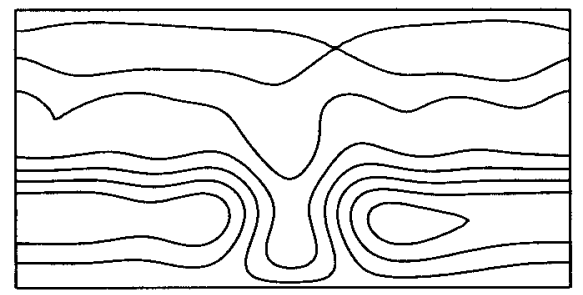

FIG. 10. Isolines of the streamfunction for the three steady states of the full nonlinear model with Gaussian topography at a topographic height of $800 \mathrm{~m}$. The upper panels show the superresonant, the middle panels the unstable resonant, and the lower panels the stable resonant state, corresponding to the ordering in Table 3 . The contour interval is $10000 \mathrm{~m}^{2} \mathrm{~s}^{-1}$ for the superresonant state and $5000 \mathrm{~m}^{2} \mathrm{~s}^{-1}$ for the two other states.

It is quite instructive to compare again the transports, momentum balances, and streamfunction patterns of the different steady states for topographic values where three steady states coexist. Figure 10 shows the steady states for a topography height of $800 \mathrm{~m}$ and Table 3 gives the corresponding integral momentum balances and transports. The first steady state, which is the superresonant one, shows a similar pattern in both layers, with an equatorward deflection of the streamlines over the topographic elevation, as would be expected in a barotropic model from the shape of the geostrophic con-

TABLE 3. Mass transports and relative size of terms in the zonal momentum balance for the three steady states of the model with Gaussian topogaphy of 800-m height, shown in Fig. 10. For a definition of the terms see Table 2 .

\begin{tabular}{cccccccc}
\hline \hline & \multicolumn{2}{c}{ Transports (Sv) } & & \multicolumn{4}{c}{ Momentum balances (\%) } \\
\cline { 2 - 3 } \cline { 5 - 8 } State & $T_{1}$ & $T_{2}$ & & IS & IF & BS & BF \\
\hline 1 & 315 & 260 & & 76 & 24 & 62 & 38 \\
2 & 144 & 44 & & 56 & 44 & 94 & 6 \\
3 & 138 & 46 & & 54 & 46 & 95 & 5 \\
\hline
\end{tabular}

tours. Over the northern flank of the ridge the current forms a relatively narrow jet, but the width of this jet should not be interpreted too much, because it is limited by the still relatively coarse resolution implied by the truncation. The flow in the lower layer is somewhat weaker than in the upper layer.

The second and third steady states, which are both near resonance, have relatively similar streamfunction patters. In the upper layer both show a northward deflection of the current over the topographic obstacle that is stronger in the second, unstable state and a little bit weaker in the third, stable state. Compared to the superresonant state the deflected current is broader and possesses a stronger asymmetry with respect to the location of the obstacle, that is, the current turns more strongly upstream than downstream of the ridge. There are two closed circulation cells in the lee of the ridge in the upper layer. Besides the generally weaker flow (note the different contour interval in Fig. 10 for the superresonant state compared to the resonant states), the main difference to the superresonant state is a southward deflection over the topography in the lower layer. This 
behavior was already found in one of the resonant states of the low-order model. The mean zonal flow in the lower layer is very small, as indicated by the small number of streamlines passing through the channel. There is an elongated closed cyclonic circulation in the southern part of the channel away from the topography.

\section{Summary and conclusions}

The analysis of eddy-resolving numerical models of channel flow as a simple analogy to the Antarctic Circumpolar Current starting with the work of MHC78 has shown bottom form stress to be the main sink of zonal momentum, balancing the momentum input by westerly winds. Krupitsky and Cane (1994) and Wang and Huang (1995) have suggested an explanation of this balance by the geometry of geostrophic contours near Drake Passage. In their barotropic analytical models they found high values of form stress when topography that extends from the northern to the southern channel boundary is high enough to disrupt all zonally reconnecting geostrophic contours. Yet, this explanation does not apply to topographic obstacles that are isolated from the boundaries, where MHC78 and WMO91 also have found a dominating role of bottom form stress.

In this paper an additional mechanism for the generation of strong bottom form stress has been studied, the resonance of baroclinic Rossby waves that are excited by the interaction of the zonal current with topography and are trapped by the counteraction of advection and wave propagation. In an analytically solvable baroclinic low-order model this mechanism has been shown to lead to the existence of different flow regimes, resonant and nonresonant or blocked and nonblocked. The resonant steady states are characterized by a barotropic mean zonal velocity near the absolute value of the baroclinic wave propagation speed. There is a range of topographic heights where blocked and nonblocked states coexist, but for high enough topographies there is only one resonant solution. In the resonant solution the zonal current in the lower layer is deflected from its zonal path against the direction of the geostrophic contours. Interfacial friction, that is, the parameterized vertical eddy transfer of momentum plays an important indirect role in the zonal momentum balances by establishing a phase difference between the barotropic and baroclinic streamfunction.

The validity of the low-order approximation has been investigated by studying numerically the steady states of a less severely truncated spectral model. Although there can be more steady states in this model for one set of parameter values than in the low-order model, the structure of all these states does not differ much from the structure of the blocked or unblocked states of the low-order model. For low and for high topographic heights there is only one steady state that corresponds closely to the low-order model state.

Finally, the existence of resonant steady states has been investigated for a topography in the form of a Gaussian ridge, a form that has been widely used in numerical channel model studies (MHC78; WMO91). Although the structure of the streamfunction fields in this model differs strongly from the one for sinusoidal topography, the steady states can also be classified as either resonant or nonresonant, sharing some qualitative features with the corresponding states for the sinusoidal topography. Besides the mean zonal velocity the main correspondence is the deviation of the current over the topography in the lower layer, which is against the sense of the geostrophic contours for the blocked states. A difference to the simple topography case is the existence of a nonresonant state over the whole range of topographic heights considered. This is due to nonlinear interactions between the wave field and the topography, as has been shown by a comparison with a quasilinear model. The existence of a nonresonant and two resonant steady states over a wider range of topographic heights for Gaussian topography might explain the observed long-period variability in numerical studies of channel flow.

Several objections can be raised against transferring results from such a simple model with idealized topography to the real ACC. Some of them are

- The low-order model descibed here is limited to isolated topography by construction, and thus cannot describe the influence of the topography near Drake Passage on the ACC. But analyses of sea surface height statistics (Gille and Kelly 1996) suggest that other topographical obstacles, such as the Kerguelen and Crozet Plateaus, could also be sources of form drag. Here Rossby wave resonance might be important.

- Unlike a channel, the Southern Ocean has no meridional boundaries, and topographically excited Rossby waves may propagate away meridionally instead of being trapped near topography. Hughes (1996), however, has demonstrated the existence of a zonal wave guide for Rossby waves in FRAM model results for the ACC, so perhaps a channel is actually not too bad to describe Rossby wave propagation in the ACC. Yet, this raises new questions, for example, on the role of critical layers.

- The ACC is probably superresonant with respect to baroclinic waves; Hughes (1996) finds eastward propagation of Rossby waves inside the ACC waveguide. But the generation of form stress by resonance is not limited to (sub)resonant flows; this may be seen in the momentum balances of the superresonant states (Tables 2 and 3 ) that have barotropic zonal velocities sufficiently near resonance to produce significant form stresses.

- The model has shown vertical eddy transfer of momentum, parameterized as interfacial friction, to be important in setting up the phase shifts of the stationary pressure anomalies against topography that are necessary for transferring momentum by form stress. 
The parameterization by an interfacial friction with a constant coefficient is equivalent to a parameterization of the lateral eddy fluxes of QPV by diffusion of interfacial height (or stretching vorticity) with a constant diffusion coefficient (Marshall 1981); yet such a parameterization contradicts the findings in eddyresolving numerical models (Olbers et al. 1999, manuscript submitted to J. Phys. Oceanogr.). We were only able to show that Rossby wave resonance is insensitive to the size of the interfacial friction parameter, and we can make no conclusions about sensitivity to the form of the parameterization.

In spite of all these problems we think that the idealized model presented here complements the analytical studies by Krupitsky and Cane (1994) and Wang and Huang (1995) by proposing an additional mechanism of form drag generation; we hope that this may prove helpful in interpreting data from the real ACC.

Acknowledgments. I thank D. Olbers for his help and for many discussions on ACC dynamics. Thanks also go to A. Hense, who had the idea to use low-order models to learn more about the dynamics in channel models of the ACC, and to V. Gryanik, who kept asking critical questions where I did not notice problems.

\section{APPENDIX}

\section{Equations of the Moderate-Order Model}

The moderate-order model differs from the low-order model in the expansion of the streamfunction fields:

$$
\begin{aligned}
& \Phi(x, y, t)=-U(t) y+\sum_{m=-M}^{M} \sum_{n=1}^{N} \phi_{m, n}(t) F_{m, n}(x, y) \\
& \Theta(x, y, t)=-u(t) y+C(t)+\sum_{m=-M}^{M} \sum_{n=1}^{N} \theta_{m, n}(t) F_{m, n}(x, y),
\end{aligned}
$$

where the maximum meridional wavenumber $N=M$ $-|m|+1$ is a function of the zonal wavenumber. Reality of the fields requires $\phi_{-m, n}=\phi_{m, n}^{*}$ and $\theta_{-m, n}=\theta_{m, n}^{*}$, with the asterisk symbolizing the complex conjugate.

Projection of the barotropic QPV balance (10) results in

$$
\begin{aligned}
0= & \tau_{0} \delta_{m}^{0} \delta_{n}^{2}-\frac{\epsilon a_{m, n}}{1+\alpha}\left(\phi_{m, n}-\alpha \theta_{m, n}\right)+\frac{i m \beta}{l} \phi_{m, n} \\
& -\frac{i m}{l}(U-\alpha u) b_{m, n} \\
& -\sum_{m^{\prime}, n^{\prime}} \sum_{m^{\prime \prime}, n^{\prime \prime}} G_{m, n}^{m^{\prime}, m^{\prime \prime}, n^{\prime}, n^{\prime \prime}}\left(\phi_{m^{\prime}, n^{\prime}}-\alpha \theta_{m^{\prime}, n^{\prime}}\right) b_{m^{\prime \prime}, n^{\prime \prime}},
\end{aligned}
$$

where $a_{m, n}=n^{2}+m^{2} / l^{2}$ and the Kronecker delta is $\delta_{a}^{b}=1$ if $a=b$ and 0 otherwise; $b_{m, n}$ are the Fourier coefficients of the topography. The stationary baroclinic balance (11) yields

$$
\begin{aligned}
0= & \tau_{0} \delta_{m}^{0} \delta_{n}^{2} \frac{1+\alpha}{\alpha}-(1+\alpha) a_{m, n} \mu \theta_{m, n} \\
& +\frac{i m \beta}{l}\left(\phi_{m, n}+\theta_{m, n}\right)-\frac{i m}{l \sigma}\left(U \theta_{m, n}-u \phi_{m, n}\right) \\
& -\frac{1}{\sigma} \sum_{m^{\prime}, n^{\prime}} \sum_{m^{\prime \prime}, n^{\prime \prime}} G_{m, n}^{m^{\prime}, m^{\prime \prime}, n^{\prime}, n^{\prime \prime}} \phi_{m^{\prime}, n^{\prime}} \theta_{m^{\prime \prime}, n^{\prime \prime}}
\end{aligned}
$$

The interaction coefficients $G_{m, n}^{m^{\prime}, m^{\prime \prime}, n^{\prime}, n^{\prime \prime}}$ describe the coupling between different modes, caused by the advective term. One finds that

$$
\begin{aligned}
G_{m, n}^{m^{\prime}, n^{\prime \prime}, n^{\prime}, n^{\prime \prime}=\frac{i}{2}} \delta_{m}^{m^{\prime}+m^{\prime \prime}} & {\left[\left(m^{\prime} n^{\prime \prime}-m^{\prime \prime} n^{\prime}\right) \delta_{n}^{n^{\prime}+n^{\prime \prime}}\right.} \\
& \left.-\left(m^{\prime} n^{\prime \prime}+m^{\prime \prime} n^{\prime}\right)\left(\delta_{n}^{n^{\prime \prime}-n^{\prime}}-\delta_{n}^{n^{\prime}-n^{\prime \prime}}\right)\right] .
\end{aligned}
$$

As in the low-order model the equations obtained by projection of (10) and (11) have to be complemented by three additional equations that determine $U, u$, and $C$. By insertion of the expansions (A1) and (A2) into the momentum and mass constraints (7) to (8) one gets

$$
\begin{aligned}
U & =\sum_{n=1}^{M+1} n \psi_{0, n} \\
u & =\sum_{n=1}^{M+1} n \theta_{0, n} \\
C & =\frac{\pi}{2} u-\frac{2}{\pi} \sum_{n=\text { odd }} \frac{\theta_{0, n}}{n} .
\end{aligned}
$$

\section{REFERENCES}

Charney, J. G., and J. G. DeVore, 1979: Multiple flow equilibra in the atmosphere and blocking. J. Atmos. Sci., 36, 1205-1216.

— libra, and propagating planetary waves in baroclinic, orographically forced, planetary wave systems. J. Atmos. Sci., 37, 11571176.

Davey, M. K., 1980: A quasi-linear theory for rotating flow over topography. Part I: Steady $\beta$-plane channel. J. Fluid Mech., 99, 267-292.

Doedel, E. J., and X. J. Wang, 1995: AUTO94: Software for continuation and bifurcation problems in ordinary differential equations. Tech. Rep. CRPC-95-2, Center for Research on Parallel Computing, California Institute of Technology. [Available from California Institute of Technology, 12000 East California Boulevard, Pasadena, CA 91125.]

Egger, J., 1978: Dynamics of blocking highs. J. Atmos. Sci., 35, 17881801.

Gille, S. T., and K. A. Kelly, 1996: Scales of spatial and temporal variability in the Southern Ocean. J. Geophys. Res., 101(C), 8759-8773.

Hughes, C. W., 1996: The Antarctic Circumpolar Current as a waveguide for Rossby waves. J. Phys. Oceanogr., 26, 1375-1387.

Krupitsky, A., and M. A. Cane, 1994: On topographic pressure drag in a zonal channel. J. Mar. Res., 52, 1-23.

_ , V. M. Kamenkovich, N. Naik, and M. A. Cane, 1996: A linear equivalent barotropic model of the Antarctic Circumpolar Current with realistic coastlines and bottom topography. J. Phys. Oceanogr., 26, 1803-1824.

Marshall, J., 1981: On the parameterization of geostrophic eddies in the ocean. J. Phys. Oceanogr., 11, 257-271. 
D. Olbers, H. Ross, and D. Wolf-Gladrow, 1993: Potential vorticity constraints on the dynamics and hydrography of the Southern Ocean. J. Phys. Oceanogr., 23, 465-487.

McWilliams, J. C., 1977: A note on a consistent quasigeostrophic model in a multiply connected domain. Dyn. Atmos. Oceans, 1, 427-441.

- W. R. Holland, and J. H. S. Chow, 1978: A description of numerical Antarctic Circumpolar Currents. Dyn. Atmos. Oceans, 2, 213-291.

Munk, W. H., and E. Palmén, 1951: Note on the dynamics of the Antarctic Circumpolar Current. Tellus, 3, 53-55.

Olbers, D., 1998: Comments on "On the obscurantist physics of 'form drag' in theorizing about the Circumpolar Current." J. Phys. Oceanogr., 28, 1647-1654.

— zonal flows. Decadal Climate Variability, D. L. T. Anderson and J. Willebrand, Eds., NATO ASI series I 44, Springer Verlag, 407-443.

Parker, T. S., and L. O. Chua, 1989: Practical Numerical Algorithms for Chaotic Systems. Springer Verlag, 348 pp.

Pedlosky J., 1981: Resonant topographic waves in barotropic and baroclinic flows. J. Atmos. Sci., 38, 2626-2641.

Stevens, D. P., and V. O. Ivchenko, 1997: The zonal momentum balance in an eddy resolving general circulation model of the Southern Ocean. Quart. J. Roy. Meteor. Soc., 123, 929-951.

Treguier, A. M., and J. C. McWilliams, 1990: Topographic influences on wind-driven, stratified flow in $\beta$-plane channel: An idealized model for the Antarctic Circumpolar Current. J. Phys. Oceanogr., 20, 321-343.

Tung, K. K., and A. J. Rosenthal, 1985: Theories of multiple equilibria-A critical reexamination. Part I: Barotropic models. $J$. Atmos. Sci., 42, 2804-2819.

Völker, C., 1996: Barokline Strömung über periodischer Topographie: Untersuchungen an analytischen und numerischen Modellen. Ph.D. thesis, Universität Bremen, 65 pp.

Wang, L., and R. X. Huang, 1995: A linear homogeneous model of wind-driven circulation in a $\beta$-plane channel. J. Phys. Oceanogr., 25, 587-603.

Wolff, J.-O., and D. Olbers, 1989: The dynamical balance of the Antarctic Circumpolar Current studied with an eddy resolving quasigeostrophic model. Mesoscale/Synoptic Coherent Structures in Geophysical Turbulence, J. C. J. Nihoul and B. M. Jamart, Eds., Elsevier Science, 435-458.

— topography in a zonal $\beta$ plane channel: A quasi-geostrophic model of the Antarctic Circumpolar Current. J. Phys. Oceanogr., 21, 236-264. 